Methods: A retrospective observational study was conducted including patients with RA treated in a tertiary hospital between June 2006 and May 2017 who had received at least one RTX cycle. At RTX initiation we analysed: age, sex, comorbidities and Charlson score, disease duration, presence of rheumatoid factor (RF)/anti-citrullinated protein antibodies (ACPA), disease activity (DAS28), acute phase reactants (CRP, ESR), previous biological treatments; concomitant treatment (csDMARD/glucocorticoids (GC)). Serum Ig levels before every RTX cycle, the number of RTX cycles and adverse events (AE), including serious and opportunistic infections were also analysed.

Results: We included 53 patients ( $86.8 \%$ women, mean age $55.5 \pm 13.5$ years), $58 \%$ with a Charlson score $>3$. Mean disease duration was $16 \pm 9.1$ years; $84.9 \%$ and $92.5 \%$ were RF and ACPA positive, respectively.

Before starting RTX, $81 \%$ of patients had received other biologic drugs $(58.5 \% \geq$ $2), 88 \%$ received concomitant csDMARD, (52\% methotrexate and $32 \%$ leflunomide) and $81 \%$ were treated with $\mathrm{GC}$ (median dose $10 \mathrm{mg}, \mathrm{P}_{25-75} 5-10 \mathrm{mg}$ ). The median number of RTX cycles received per patient was $5\left(P_{25-75} 2-6\right)$.

80 AE were reported: 12 infusion reactions, 8 cases of neutropenia, 51 infections (18 respiratory, 8 urinary, 4 skin and soft tissues, 8 gastrointestinal, 4 cases of non-disseminated herpes zoster, 1 bacteremia, 2 septic shock and 6 other) of which 19 were serious. and 5 malignancies $(2$ melanomas, 2 cervix, and 1 bladder) were also notified. No opportunistic infections were reported.

lg levels were obtained for 41 subjects: 7,5 and 1 patients had low levels of lgG, $\lg M$ and $\lg A$, respectively.

Patients who developed infections received a greater number of RTX cycles $(p<0.0002)$ and had more frequently low levels of serum lgG during follow-up $(p<0.044)$ than those who did not have infections.

Conclusions: Long-term exposure to RTX showed a good safety profile with a low incidence of serious infectious and no opportunistic infections.

Factors associated with the development of infections were the number of cycles received and low serum levels of lgG at any point during follow-up.

Acknowledgements: The authors would like to thank Dr. García de Yébenes who provided statistical support

Disclosure of Interest: None declared

DOI: 10.1136/annrheumdis-2018-eular.3634

\section{AB0467 $\quad$ SUSTAINED CLINICAL RESPONSE IN REFRACTORY RHEUMATOID ARTHRITIS PATIENTS WITH A LOW- DOSE RITUXIMAB RETREATMENT REGIMEN}

S.D.L.C. Rodriguez García, R. Castellanos-Moreira, M.V. Hernandez-Miguel, A. Cuervo, V. Ruiz-Esquide, J. Ramirez, O. Camacho, J.D. Cañete, J. Gomez Puerta, R. Sanmartí. Rheumatology, Hospital Clinic de Barcelona, Barcelona, Spain

Background: The standard dose of rituximab (RTX) in rheumatoid arthritis (RA) is two intravenous (iv) $1 \mathrm{~g}$ infusions, separated by two weeks. Recently, the efficacy of a low-dose of RTX for retreatment in RA patients has been reported. ${ }^{1}$

Objectives: Our aim was to assess the long-term sustained effectiveness of a low-dose of RTX in daily clinical practice.

Methods: Observational retrospective study including all RA patients treated on a tertiary hospital who had received at least one cycle of RTX, at the standard dose, between June 2006 and May 2017. We selected those patients who achieved a good or moderate EULAR response and thereafter were down-titrated to a low-dose regimen $(1 \mathrm{~g})$. Variables analysed: age, sex, disease duration, presence of ACPA (antiCCP2) and rheumatoid factor (RF), glucocorticoid (GC) and conventional synthetic DMARD (csDMARD) use and dosage before and after RTX treatment, number of biologic DMARD (bDMARD) used prior to initiating RTX. Disease activity was measured using DAS28 index (prior to first RTX infusion, at low-dose regimen initiation and at last follow-up visit)

Results: 53 patients received, at least, one cycle of $2 \mathrm{~g} \mathrm{RTX}, 70 \%$ achieved a good or moderate EULAR response and were stepped-down to a low dose retreatment regimen. Baseline characteristics of patients receiving low-dose RTX were: mean age $56.4 \pm 10.9$ years; $13.5 \%$ male, mean disease duration $12.7 \pm 9.8$ years, $91.9 \%$ RF +and $97.3 \%$ ACPA +; mean DAS28 prior to RTX initiation 5,79 $\pm 1,17$.

$73 \%$ of patients had received other bDMARD before RTX $48 \% 2$ or more. $92 \%$ were on cs-DMARDs, $51.4 \%$ methotrexate (MTX) and $37.8 \%$ leflunomide (LEF) and $86.5 \%$ were receiving concomitant $\mathrm{GC}$ (median dose $10 \mathrm{mg}, \mathrm{P}_{25-75} 5-10$ $\mathrm{mg}$ ). $73 \%$ of subjects received only one standard cycle before RTX dose reduction.
Mean DAS28 decreased significantly between the first visit on $1 \mathrm{~g}$ RTX vs the last follow-up visit (4.08 vs 3.04; $\mathrm{p}<0.0001$ ). Additionally, 11 patients (8 MTX, 3 LEF) were able to reduce csDMARD dosage, $56.3 \%$ of patients receiving GC at the initiation of low-dose retreatment were able to reduce the dose (median $10 \mathrm{mg}$ vs $5 \mathrm{mg} ; \mathrm{p}<0.0001$ ), and $28 \%$ discontinued GC therapy

After a mean follow-up of $3 \pm 1.8$ years, RTX was withdrawn in 10 patients: 8 due to adverse events (recurrent infections in 4 ) and 2 cases due to loss of efficacy.

Conclusions: A sustained clinical response was observed with the 1 gr retreatment of RTX after a long-term follow-up period.

\section{REFERENCE:}

[1] Mariette X, et al. Ann Rheum Dis 2014;73:1508-14.

Disclosure of Interest: None declared

DOI: 10.1136/annrheumdis-2018-eular.4008

\section{AB0468 CLINICAL AND ULTRASONOGRAPHIC EFFECTIVENESS IN TWO COHORTS OF RHEUMATOID ARTHRITIS PATIENTS TREATED WITH ABATACEPT: A REAL LIFE STUDY}

S. Parisi, M.C. Ditto, M. Scarati, M. Priora, C.L. Peroni, E. Fusaro. Rheumatology Unit, AOU Città della Salute e della Scienza di Torino, Turin, Italy

Background: Synovitis in Rheumatoid Arthritis (RA) is a phenomenon related to the development of erosions and progressive structural damage; early synovitis improvements are successfully associated with long-term clinical and structural outcomes.

Objectives: The aim of this study was to evaluated the efficacy of abatacept in two cohort of patients treated with Abatacept as the first and second or third line of treatment.

Methods: We evaluated patients affected by RA (according to ACR 2010 criteria) and were divided into two groups:

Group A: patients with moderate or severe active RA, non-responders to Metothrexate (MTX), bDMARDs naïve, treated with Abatacept 125 mg/wk;

Group B: patients with moderate or severe active RA, non-MTX and anti-TNF responders, treated with Abatacept $125 \mathrm{mg} / \mathrm{wk}$;

The concomitant treatment with MTX was maintained unchanged in those patients who were taking it at stable doses before the start of the study (10$15 \mathrm{mg} /$ week for $>28$ days); concomitant therapies such as low-dose systemic CS (prednisone $\leq 7.5 \mathrm{mg} / \mathrm{day}$ ) and NSAIDs have been maintained for at least 4 weeks if stable. The activity of RA was calculated with the DAS28-CRP according to the clinical practice protocol (week $0,4,12,24$ ). The Ultrasound (US) evaluation of the synovitis was done according to the Omeract criteria (Grey Scale and PDUS score: 0 to 3 )

Results: We recruited consecutively 34 patients with RA, 16 pts (male $n=4$; $25,00 \%$ ) took Abatacept as the first line (Group A), and 18 pts (males $n=5$; $27.70 \%$ ) took Abatacept as followed by another anti-TNF drugs (Group B). The mean age was $57.2 \pm 10.7$ years (median 60 , range $45-72$ ); mean of DAS28 at baseline was $4.8 \pm 0.9$ (median 4.7; range 3.9-5.6); mean duration of the disease was $15.3 \pm 5.7$ years (median 10; range $3-22$ ). Tab. 1

A constant improvement of the DAS28 score is shown in both groups examined until the end of the follow up, resulting respectively $-\Delta 2.0$ for Group $A(p<0.05)$ and $-\Delta 2.1(p<0.05)$ for Group B. The total PDUS score decreased in both groups from week 4 , with a mean change $(95 \% \mathrm{Cl}$ ) compared to baseline of -0.8 (range $-1.4 /-0.2$ ) and progressive mean significant improvement until follow-up (Gr.A $p<0.05$; Gr.B $p<0.05$ ). No serious adverse events or infections were observed Patients with ACPA positive showed a greater improvement trend compared to other patients in both groups ( $p: 0,068)$. Figure 1

Abstract AB0468 - Table 1. Cohort of patients at baseline

\begin{tabular}{|l|c|c|}
\hline \multicolumn{1}{|c|}{ Characteristics } & Group A (Abatacept 1 $^{\text {th }}$ line) & Group B (Abatacept 2 $^{\text {sd }}$ or $3^{\text {rd }}$ line) \\
\hline Patients (n. $)$ & 16 & 18 \\
\hline Age (mean \pm SD), yy & $44,20( \pm 8,7)$ & $56,10( \pm 10,6)$ \\
\hline Female (\%) & $76,00 \%$ & $77,00 \%$ \\
\hline Disaese Duration (mean $\pm S D), y y$ & $7,60( \pm 5,3)$ & $12,70( \pm 5,6)$ \\
\hline DAS28-CRP (mean $\pm S D)$ & $5,10( \pm 0,9)$ & $4,90( \pm 1,1)$ \\
\hline PDUS score (mean $\pm S D)$ & $12,30( \pm 2,5)$ & $12,60( \pm 3,1)$ \\
\hline
\end{tabular}

